\title{
Diagonally Compressed TNAZ - A DFT Treatment
}

\section{Lemi Türker}

Department of Chemistry, Middle East Technical University, Üniversiteler, Eskişehir Yolu No: 1, 06800 Çankaya/Ankara, Turkey; e-mail: 1turker@gmail.com; lturker@metu.edu.tr

\begin{abstract}
TNAZ is an insensitive explosive material having a 4-membered azetidine ring system which has three nitro groups substituted, one of them is a nitramine type. In the present density functional treatise at the level of B3LYP/6-311++G(d,p), the 4-membered ring of TNAZ is compressed diagonally either along the $\mathrm{X}$ - or $\mathrm{Y}$-axis direction. Various properties (including energies, quantum chemical and spectral etc.) in the perturbed systems have been searched and discussed.
\end{abstract}

\section{Introduction}

An energetic small-ring compound 1,3,3-trinitroazetidine, also known as TNAZ, is the most widely studied (theoretically and experimentally) explosive recently [1-4] because of continuous research to get more powerful but insensitive explosives. It is a highly nitrated four membered nitrogen heterocyclic ring having $\mathrm{N}-\mathrm{NO}_{2}$ and $\mathrm{C}-\mathrm{NO}_{2}$ groups. It possesses improved performance in comparison to conventional melt castable explosive trinitrotoluene (TNT). The presence of small strained ring system contributes additional energy [5-10]. In the literature there are various methods reported for the synthesis of 1,3,3-trinitroazetidine [11].

TNAZ has been proposed as potential replacement for TNT due to its high performance, melt castable properties [12]. The low melting point of TNAZ $\left(101^{\circ} \mathrm{C}\right)$ enables the processing of formulations on modified production lines. Its performance is approximately $30 \%$ greater than TNT. TNAZ shows excellent thermal stability $\left(>180^{\circ} \mathrm{C}\right)$

Received: April 19, 2021; Accepted: May 18, 2021

Keywords and phrases: TNAZ, 1,3,3-trinitroazetidine, explosive, impact sensitivity, DFT.

Copyright (C) 2021 Lemi Türker. This is an open access article distributed under the Creative Commons Attribution License, which permits unrestricted use, distribution, and reproduction in any medium, provided the original work is properly cited. 
[13] besides its many added advantages over known explosives. TNAZ is a highly energetic material (more powerful than RDX) but less vulnerable than most other nitramines [14,15]. TNAZ is soluble in molten TNT (unlike HMX) and is compatible with aluminum, steel, brass and glass [16-18]. By using the pressure DSC method, the compatibility of 1,3,3-trinitroazetidine (TNAZ) with various energetic components and inert materials of solid propellants was studied [19]. On the other hand, desensitization of TNAZ via molecular structure modification has been investigated theoretically [20].

By means of molecular dynamics simulation with the ReaxFF/lg reactive force field, reactive molecular dynamics simulations of the thermal decomposition mechanism of 1,3,3-trinitroazetidine has been studied where the thermal decomposition of TNAZ crystals at high temperature was calculated [21]. Thus, the authors managed to analyzed all of the change in the potential energy of TNAZ, the formation of small-molecule products and clusters, and the initial reaction path of TNAZ. The kinetic parameters of different reaction stages in thermal decomposition of TNAZ were obtained [21].

In the present study, the azetidine ring of TNAZ molecule is compressed diagonally within the constraints of density functional theory (DFT) and the effect of perturbation on various properties of perturbed TNAZ systems have been investigated.

\section{Method of Calculation}

The initial geometry optimizations of all the structures leading to energy minima were achieved by using MM2 method followed by semi-empirical PM3 self-consistent fields molecular orbital (SCF MO) method [22,23] at the restricted level [24,25]. Subsequent optimizations were achieved at Hartree-Fock level using various basis sets. Then, geometry optimizations were managed within the framework of density functional theory $[26,27]$ at the level of $6-311++\mathrm{G}(\mathrm{d}, \mathrm{p})[24,28]$. The exchange term of B3LYP consists of hybrid Hartree-Fock and local spin density (LSD) exchange functions with Becke's gradient correlation to LSD exchange [27,29]. The correlation term of B3LYP consists of the Vosko, Wilk, Nusair (VWN3) local correlation functional [30] and Lee, Yang, Parr (LYP) correlation correction functional [31]. The vibrational analyses were also done. The total electronic energies are corrected for the zero point vibrational energy (ZPE). The normal mode analysis for each structure yielded no imaginary frequencies for the $3 N-6$ vibrational degrees of freedom, where $N$ is the number of atoms in the system. This indicates that the structure of each molecule corresponds to at least a local minimum 
on the potential energy surface. All these calculations were done by using the Spartan 06 package program [32].

\section{Results and Discussion}

In the present study, the compression of azetidine ring of TNAZ molecule has been achieved by decreasing the interatomic distance along the $\mathrm{X}$ - or $\mathrm{Y}$-axis of the molecule. In TNAZ molecule the nitramine group and two of the $\mathrm{C}-\mathrm{NO}_{2}$ groups lie along one of the diagonals of azetidine deltoid which is the longest axis of the molecule. It is called the $\mathrm{X}$ axis (see Figure 1). The other diagonal passing through the methylene carbons is labeled as $\mathrm{Y}$-axis. Figure 1 shows the axes of TNAZ molecule.

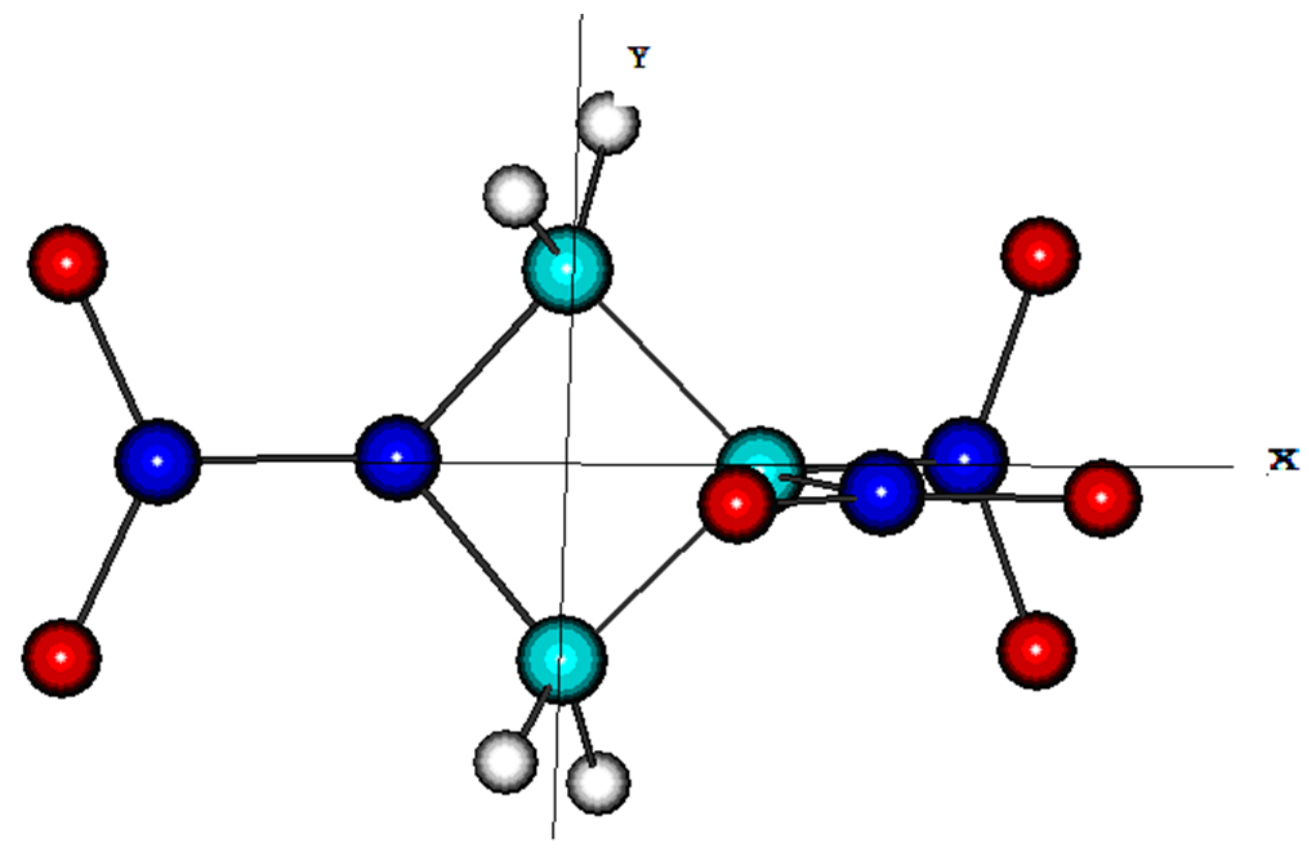

Figure 1. Axes of TNAZ molecule.

Table 1 shows the variations of diagonal interatomic distances as the molecule is compressed along the axes indicated. The azetidine ring system acts as a pantograph and as it is compressed along one of the diagonals consequently the other diagonal distance elongates. Table also includes the dipole moments as the perturbation occurs. In the table TNAZ-5 system stands for the decomposed TNAZ molecule which possesses highly elongated $\mathrm{C}-\mathrm{C}$ or $\mathrm{N}-\mathrm{NO}_{2}$ (or both) bond lengths. 
Table 1. The diagonal interatomic distances as the molecule is the compressed along the indicated direction $(\AA)$.

Compression along the $\mathrm{X}$-direction Compression along the $\mathrm{Y}$-direction

\begin{tabular}{lcccccc}
\hline System & $\begin{array}{c}\text { Dipole } \\
\text { moment }\end{array}$ & $\mathrm{X}$ & $\mathrm{Y}$ & $\begin{array}{c}\text { Dipole } \\
\text { moment }\end{array}$ & $\mathrm{X}$ & $\mathrm{Y}$ \\
\hline TNAZ & & 2.09 & 2.17 & & 2.09 & 2.17 \\
TNAZ-1 & 0.83 & 1.90 & 2.20 & 0.80 & 2.12 & 1.90 \\
TNAZ-2 & 1.45 & 1.80 & 2.22 & 1.04 & 2.15 & 1.80 \\
TNAZ-3 & 1.97 & 1.70 & 2.24 & 1.19 & 2.20 & 1.70 \\
TNAZ-4 & 2.51 & 1.60 & 2.26 & 1.24 & 2.26 & 1.60 \\
TNAZ-5 & 5.80 & 1.00 & 2.77 & 0.56 & 5.04 & 1.00 \\
\hline
\end{tabular}

Dipole moments in debye units.

Figures 2 and 3 show the optimized structures of the systems of compressed TNAZ molecule. TNAZ molecule withstands the compression in TNAZ-1 through TNAZ-4 systems undergoing merely some small perturbations, mostly conformational in character. Although, behavior of the system has not been studied beyond $1.60 \AA$ diagonal distance, at $1.00 \AA$ the system breaks down to produce TNAZ-5. Note that the change of direction of dipole moment vectors in the compressed systems with respect to its direction in TNAZ molecule. The compression causes substantial variation in charge distribution and bond lengths, thus affecting the dipole moment values (Table 1). Also note that in the present treatment the compression is rather hypothetical and confined to the azetidine ring system along the $\mathrm{X}$ - or $\mathrm{Y}$-axis only, not the compression on the whole molecule.

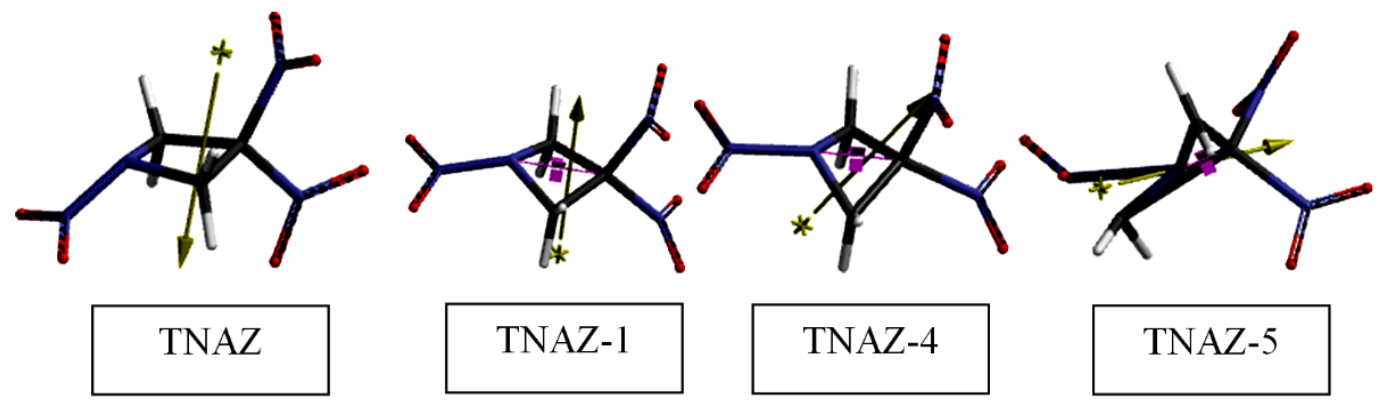

Figure 2. Optimized structures of compressed TNAZ along the X-direction. 

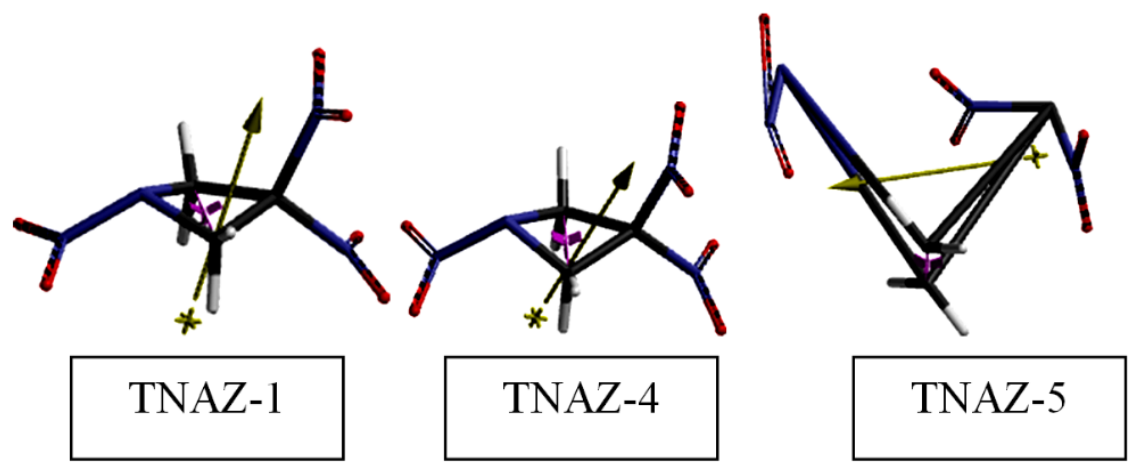

Figure 3. Optimized structures of compressed TNAZ along the Y-direction.

Table 2 shows some calculated bond lengths in the compressed TNAZ systems. As seen in the table as the diagonal distance along the $\mathrm{X}$ - (or Y-direction) decreases the $\mathrm{N}$ $\mathrm{NO}_{2}$ bond length increases in TNAZ-1 through TNAZ-4. TNAZ-5 system is exceptional due to the occurrence of bond rupture. On the other hand, $\mathrm{C}-\mathrm{NO}_{2}$ bond lengths irregularly vary in TNAZ-1 to TNAZ-5, because they are more apt to conformational changes. The two of $\mathrm{N}-\mathrm{CH}_{2}$ (or $\mathrm{C}-\mathrm{CH}_{2}$ ) bond lengths are equivalent in TNAZ through TNAZ-4. As for the bond angles of the azetidine ring, the compression obviously causes variations in them but not a regular trend is observed.

Table 2. Some calculated bond lengths $(\AA)$ in the compressed TNAZ systems.

\begin{tabular}{|c|c|c|c|c|}
\hline & \multicolumn{2}{|c|}{ Compression along the $\mathrm{X}$-direction } & \multicolumn{2}{|c|}{ Compression along the $\mathrm{Y}$-direction } \\
\hline & $\mathrm{N}-\mathrm{NO}_{2}$ & $\mathrm{C}-\mathrm{NO}_{2}$ & $\mathrm{~N}-\mathrm{NO}_{2}$ & $\mathrm{C}-\mathrm{NO}_{2}$ \\
\hline TNAZ & 1.396 & $1.523,1.532$ & 1.396 & $1.523,1.532$ \\
\hline TNAZ-1 & 1.401 & $1533,1.554$ & 1.420 & $1.510,1.533$ \\
\hline TNAZ-2 & 1.417 & $1.545,1.558$ & 1428 & $1.505,1.527$ \\
\hline TNAZ-3 & 1.443 & $1.556,1.571$ & 1.436 & $1.502,1.522$ \\
\hline TNAZ-4 & 1.490 & $1.547,1.622$ & 1.444 & $1.498,1.515$ \\
\hline TNAZ-5 & 3.293 & $1.634,1.714$ & 1.167 & $1.430,1.442$ \\
\hline
\end{tabular}

Figure 4 shows the electrostatic potential charges (ESP). Note that the ESP charges are obtained by the program based on a numerical method that generates charges that reproduce the electrostatic potential field from the entire wavefunction [32]. 
Inspection of Figures 4 and 5 indicates that the perturbation occurred on the azetidine ring affects the ESP charges on the nitro groups as well. The compression along the Xaxis decreases (in absolute value) the charges on the nitramine moiety in TNAZ-1 to TNAZ-4. The effect on C-NO 2 groups in each system is less influential and not in parallel fashion for those nitro groups. The compression in the Y-direction decreases (in absolute value) the charges on oxygen atoms of the nitramine moiety but increases the charge on the nitro group nitrogen. The effect on the other charges is not regular TNAZ-1 through TNAZ-4.

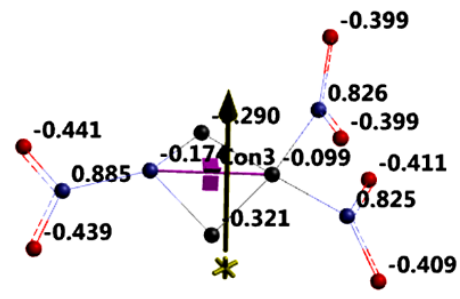

TNAZ-1
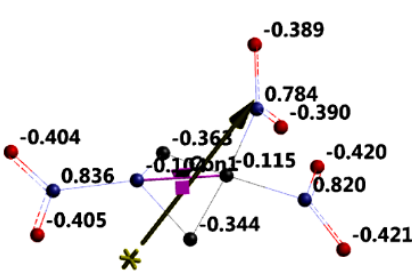

TNAZ-4

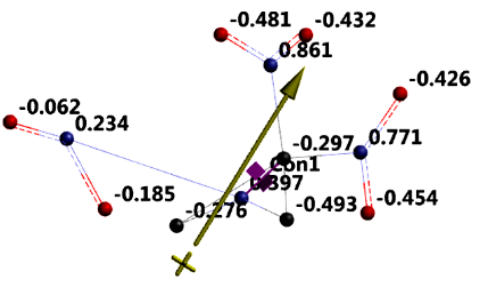

TNAZ-5

Figure 4. The ESP charges on the atoms of some compressed (along the X-direction) TNAZ systems (Hydrogens omitted).
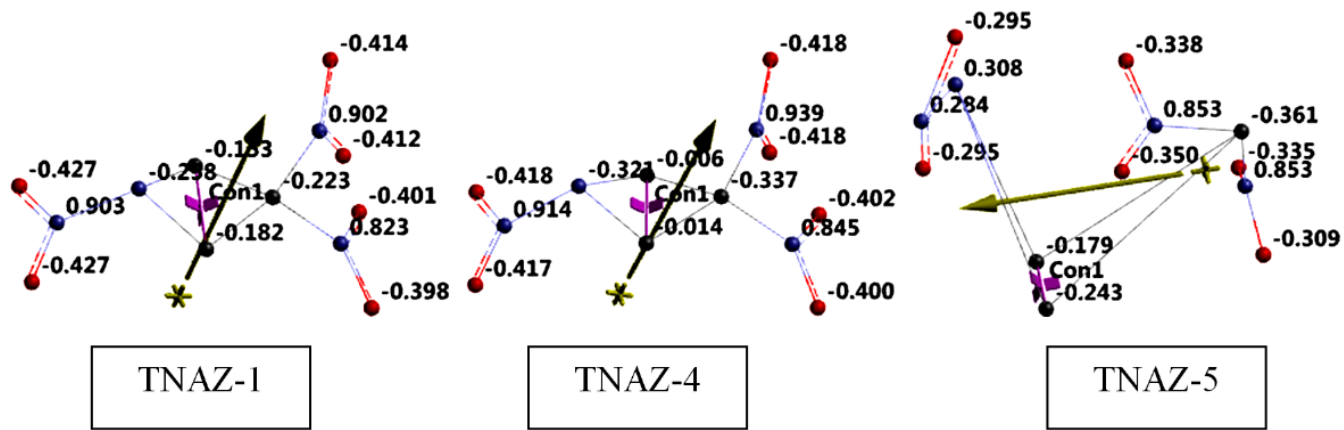

TNAZ-5

Figure 5. The ESP charges on the atoms of some compressed (along the Y-direction) TNAZ systems (Hydrogens omitted).

Table 3 displays some energies of the compressed TNAZ systems, where E, ZPE and $E_{C}$ are the total electronic energy, zero-point vibrational energy and the corrected the total electronic energy, respectively. The data indicate that as the azetidine ring is compressed in the X-direction, the perturbed systems become less and less stable electronically. The same statement holds for the compression in the Y-direction, however the compression this time is more influential to unstabilize the structures. 
Table 3. The HOMO, LUMO energies and $\Delta \varepsilon$ values of the compressed TNAZ systems.

\begin{tabular}{ccccc}
\hline $\begin{array}{c}\text { Direction of } \\
\text { compression }\end{array}$ & Structure & $\mathbf{E}$ & $\mathbf{Z P E}$ & $\mathbf{E}_{\mathbf{C}}$ \\
\hline & & & & \\
\hline $\mathbf{X}$ & TNAZ & -2066133.03 & 278.29 & -2065854.74 \\
& TNAZ-1 & -2066103.78 & 278.90 & -2065824.88 \\
& TNAZ-2 & -2066064.14 & 278.78 & -2065785.36 \\
& TNAZ-3 & -2066006.13 & 276.95 & -2065729.18 \\
& TNAZ-4 & -2065930.19 & 273.94 & -2065656.25 \\
& TNAZ-5 & -2065559.33 & 269.37 & -2065289.96 \\
\hline & & & & \\
& TNAZ-1 & -2066056.97 & 280.87 & -2065776.1 \\
& TNAZ-2 & -2065992.15 & 281.38 & -2065710.77 \\
& TNAZ-3 & -2065903.61 & 280.98 & -2065622.63 \\
& TNAZ-4 & -2065791.21 & 279.14 & -2065512.07 \\
& TNAZ-5 & -2065018.54 & 250.08 & -2064768.46 \\
\hline
\end{tabular}

Energies in $\mathrm{kJ} / \mathrm{mol}$.

Figures 6 and 7 display the calculated IR spectra of perturbed TNAZ systems which arose after the compression of the azetidine ring in the X- or Y-direction. In Figure 6 TNAZ-1 and TNAZ-4 spectra, in great extent, resemble the spectrum of TNAZ molecule. In the case of TNAZ-3, the peak at $1662 \mathrm{~cm}^{-1}$ is the symmetrical N-O stretching vibration of $\mathrm{C}-\mathrm{NO}_{2}$ groups overlapped with each other. In the other systems two of the $\mathrm{NO}_{2}$ groups on the same carbon vibrate slightly at different positions due to some conformational differences. In the case of TNAZ-5 (decomposed structure) a new peak arises at $3643 \mathrm{~cm}^{-}$ ${ }^{1}$ which is a coupled peak of $\mathrm{C}-\mathrm{NO}_{2}$ groups and the ring vibration.

In Figure 7 calculated IR spectra of some compressed (along the Y-direction) TNAZ systems are shown. In the figure the peaks at $1657-1309 \mathrm{~cm}^{-1}$ are various N-O stretchings of $\mathrm{NO}_{2}$ groups linked to the methylene group. The sharp peak at $1656 \mathrm{~cm}^{-1}$ stands for asymmetrical N-O stretching of $\mathrm{NO}_{2}$ groups. Asymmetric $\mathrm{N}-\mathrm{O}$ stretching of the nitramine $\mathrm{NO}_{2}$ group occurs at $1646 \mathrm{~cm}^{-1}$ (weak). In the spectrum of TNAZ-5 the peaks at $2974 \mathrm{~cm}^{-1}$ belong to $\mathrm{C}-\mathrm{H}$ stretchings. 

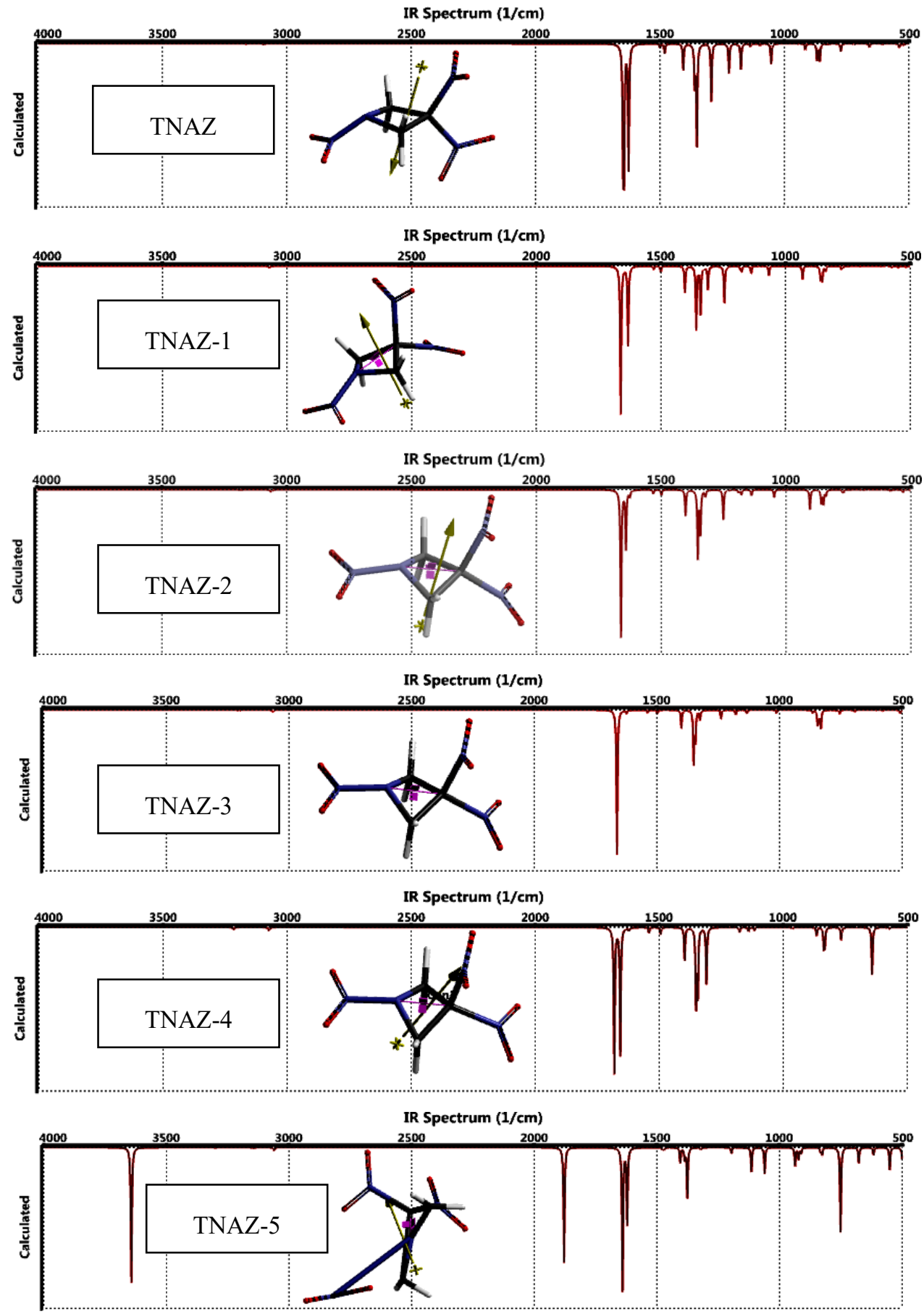

Figure 6. Calculated IR spectra of some compressed (along the X-direction) TNAZ systems. 

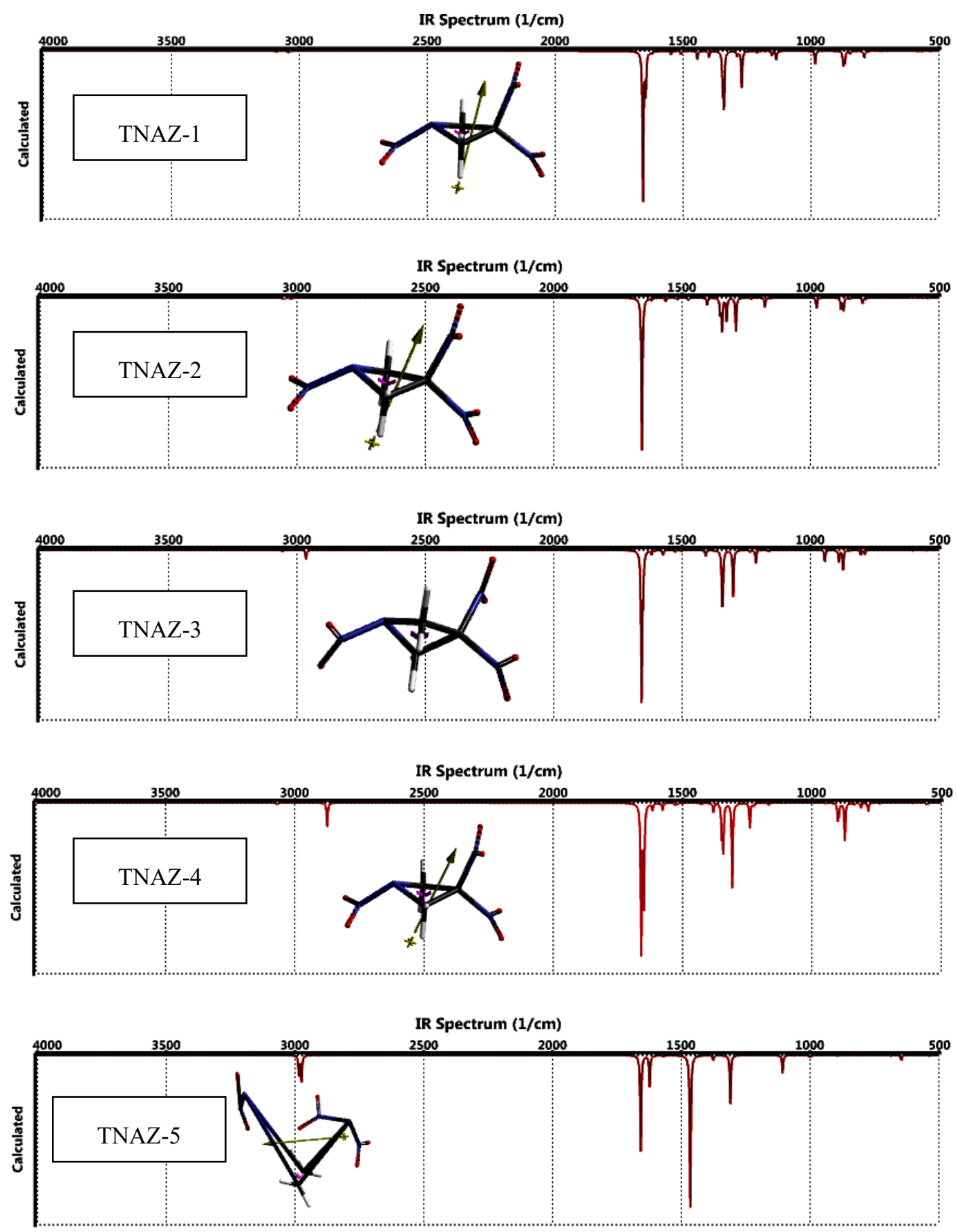

Figure 7. Calculated IR spectra of some compressed (along the Y-direction) TNAZ systems. 
Figure 8 displays some of the molecular orbital energy levels of the compressed TNAZ systems (along the X-direction). The degenerate LUMO energy levels of TNAZ decompose to distinct levels by the effect of compression. A similar behavior occurs if the compression is along the Y-direction (Figure 9). In both types of the compression the HOMO energy level raises but the LUMO energy level lowers. Also striking variations occur on the inner-lying molecular orbitals energy levels. Thermal stability is dictated by low-lying molecular orbital energy levels by both their energies and numbers [33]. As seen in the figure, as the compression develops the inner lying orbitals in the perturbed systems gradually raise up to destabilize them. Similar statements hold for the case of compression in the Y-direction (see Figure 9).
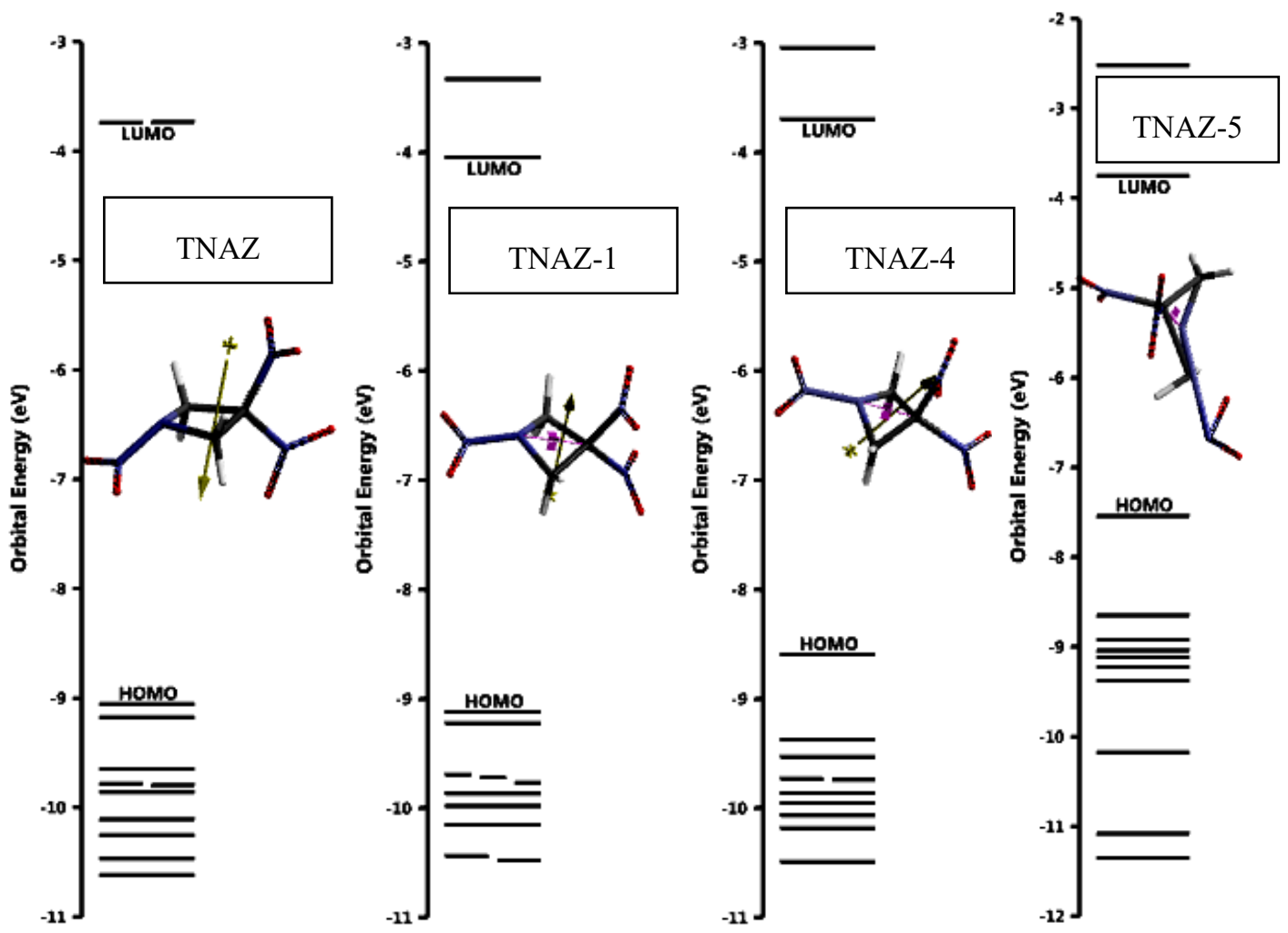

Figure 8. Some of the molecular orbital energy levels of the compressed TNAZ systems (along the X-direction). 

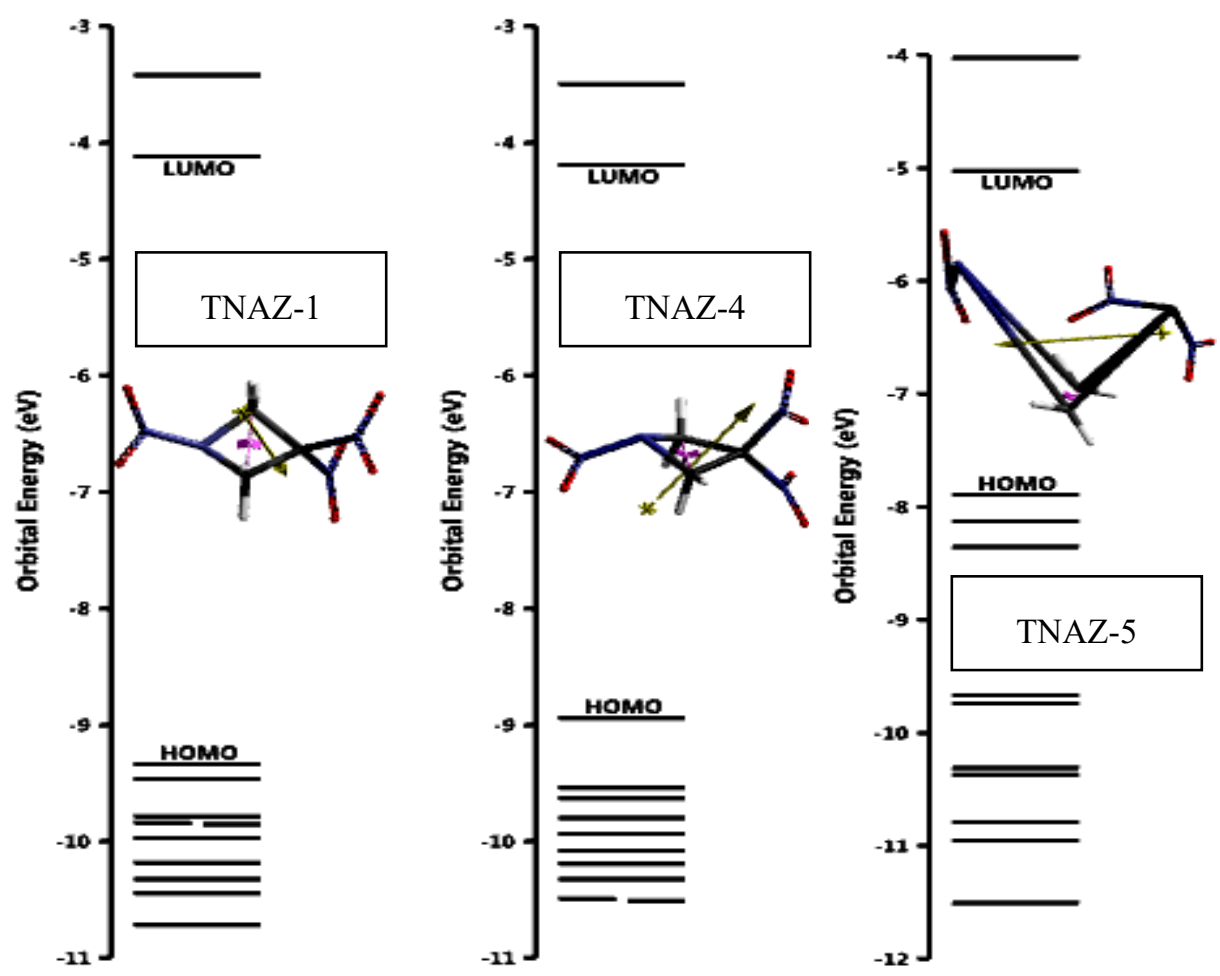

Figure 9. Some of the molecular orbital energy levels of the compressed TNAZ systems (along the Y-direction).

Table 4 lists the HOMO, LUMO energies and interfrontier molecular orbital energy gap, $\Delta \varepsilon,\left(\Delta \varepsilon=\varepsilon_{\text {номо }}-\varepsilon_{\text {LUMO }}\right)$ values of the compressed TNAZ systems. As seen in the table, the HOMO and LUMO energies raise up with small fluctuations as the compression develops in the X-direction (TNAZ-1 through TNAZ-4). The LUMO energy of TNAZ-5 is comparable to the respective value of TNAZ.

As for the compression in the Y-direction, the HOMO levels of the systems raise up with some fluctuations whereas the LUMO levels steadily get lower. The cause of all should arise from the orbital overlap interactions as the compression happens (see Figures 10 and 11).

On the other hand, sensitivity of an explosive is related to $\Delta \varepsilon$ value such that decrease of it increases the sensitivity [34,35]. Hence, the compression in either direction should increase the impact sensitivity. 
Figures 10 and 11 show the effect of compression on the HOMO and LUMO of some of the compressed TNAZ systems. As seen in Figure 10, as the compression increases the contribution of nitramine nitro group shows variation but the contribution of $\mathrm{C}-\mathrm{NO}_{2}$ groups definitely increases. Whereas in the case of the LUMO, the contribution of nitro groups increases irrespective of their kind but the nitramine nitro group is apparently overwhelming.

As for the compression along the Y-direction, the contribution of nitramine moiety in to the HOMO decreases as the compression increases but the contribution of $\mathrm{C}-\mathrm{NO}_{2}$ groups increases. On the other hand, the increasing effect of compression on the LUMO moderately increases the contribution coming from the nitramine nitro group.

Table 4. The HOMO, LUMO energies and $\Delta \varepsilon$ values of the compressed TNAZ systems.

\begin{tabular}{ccccc}
\hline $\begin{array}{c}\text { Direction of } \\
\text { compression }\end{array}$ & Structure & HOMO & LUMO & $\Delta \boldsymbol{\varepsilon}$ \\
\hline & & -873.72 & -360.76 & 512.96 \\
$\mathbf{X}$ & TNAZ & -880.03 & -390.58 & 489.45 \\
& TNAZ-1 & -879.77 & -380.86 & 498.91 \\
& TNAZ-3 & -865.78 & -370.99 & 494.79 \\
& TNAZ-4 & -829.31 & -356.77 & 472.54 \\
& TNAZ-5 & -727.73 & -361.55 & 366.18 \\
\hline & TNAZ-1 & -900.97 & -397.59 & 503.38 \\
& TNAZ-2 & -908.26 & -398.97 & 509.29 \\
& TNAZ-3 & -915.03 & -401.31 & 513.72 \\
& TNAZ-4 & -862.69 & -404.25 & 458.44 \\
& TNAZ-5 & -761.56 & -484.68 & 276.88 \\
\hline
\end{tabular}

Energies in $\mathrm{kJ} / \mathrm{mol}$.

Figure 12 shows the calculated UV-VIS spectra of some compressed (along the Xdirection) TNAZ systems. As seen in the figure, the compression along the $\mathrm{X}$-axis causes some bathochromic effect on the UV-VIS spectra of the systems considered. The underlying reason for it is that the compression decreases the HOMO-LUMO energy gap 
(see Figure 8 and Table 4). Hence, excitations of an electron to upper energy level is less energy demanding. Note that as the compression progress, a new maximum (as a shoulder) starts to improve and three of those shoulders emerge in TNAZ-5 (decomposed structure). It indicates the presence of new favorable transitions besides the HOMOLUMO transition.

Figure 13 is the calculated UV-VIS spectra of some compressed (along the Ydirection) TNAZ systems. This time compression is less effective and only a small bathochromic shift to longer wavelengths occurs from TNAZ-1 to TNAZ-4, but in the case of TNAZ-5 an extensive bathochromic shift happens with new maximum values.
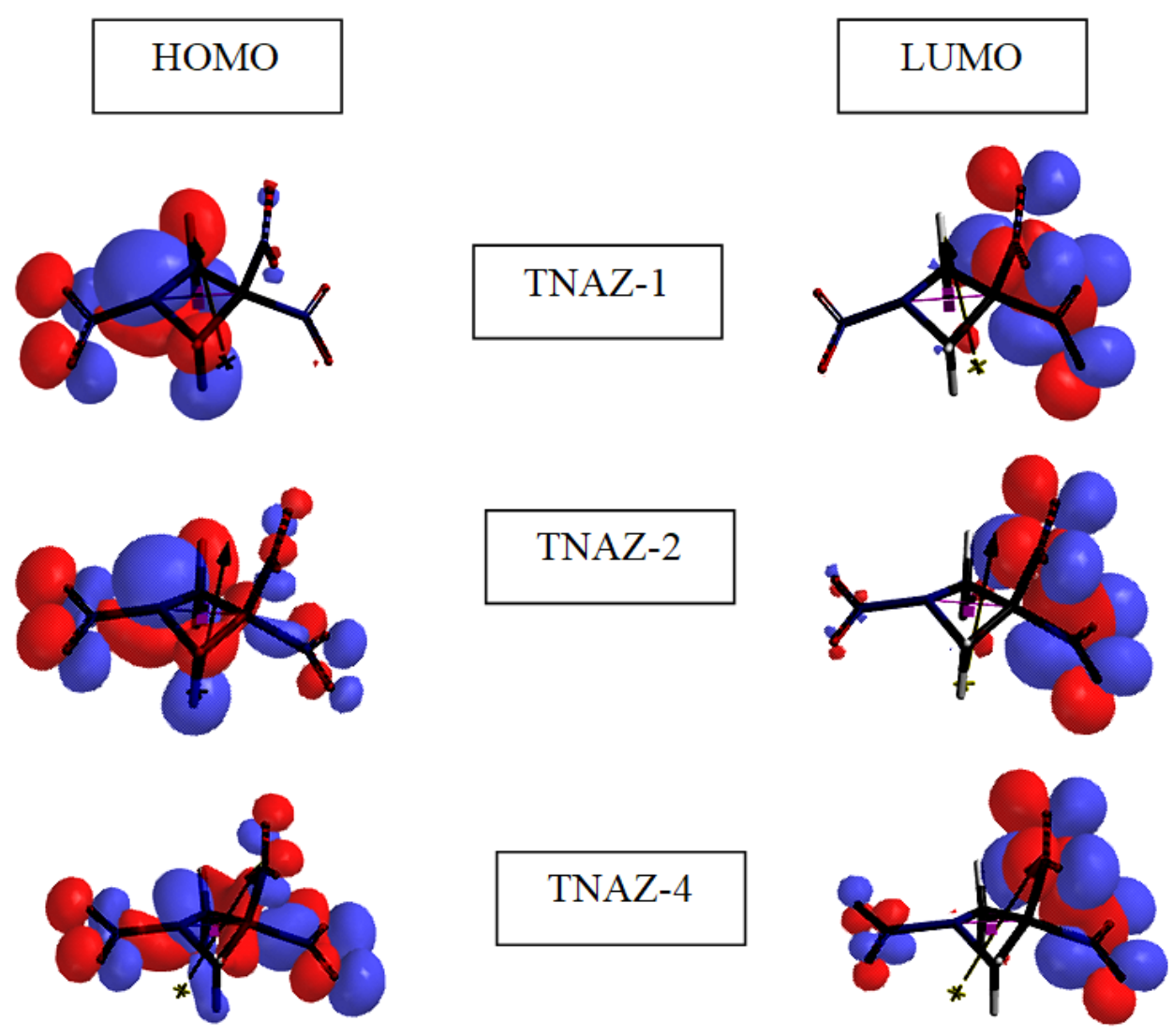

Figure 10. The HOMO and LUMO of some of the compressed TNAZ systems (along the $\mathrm{X}$-direction). 

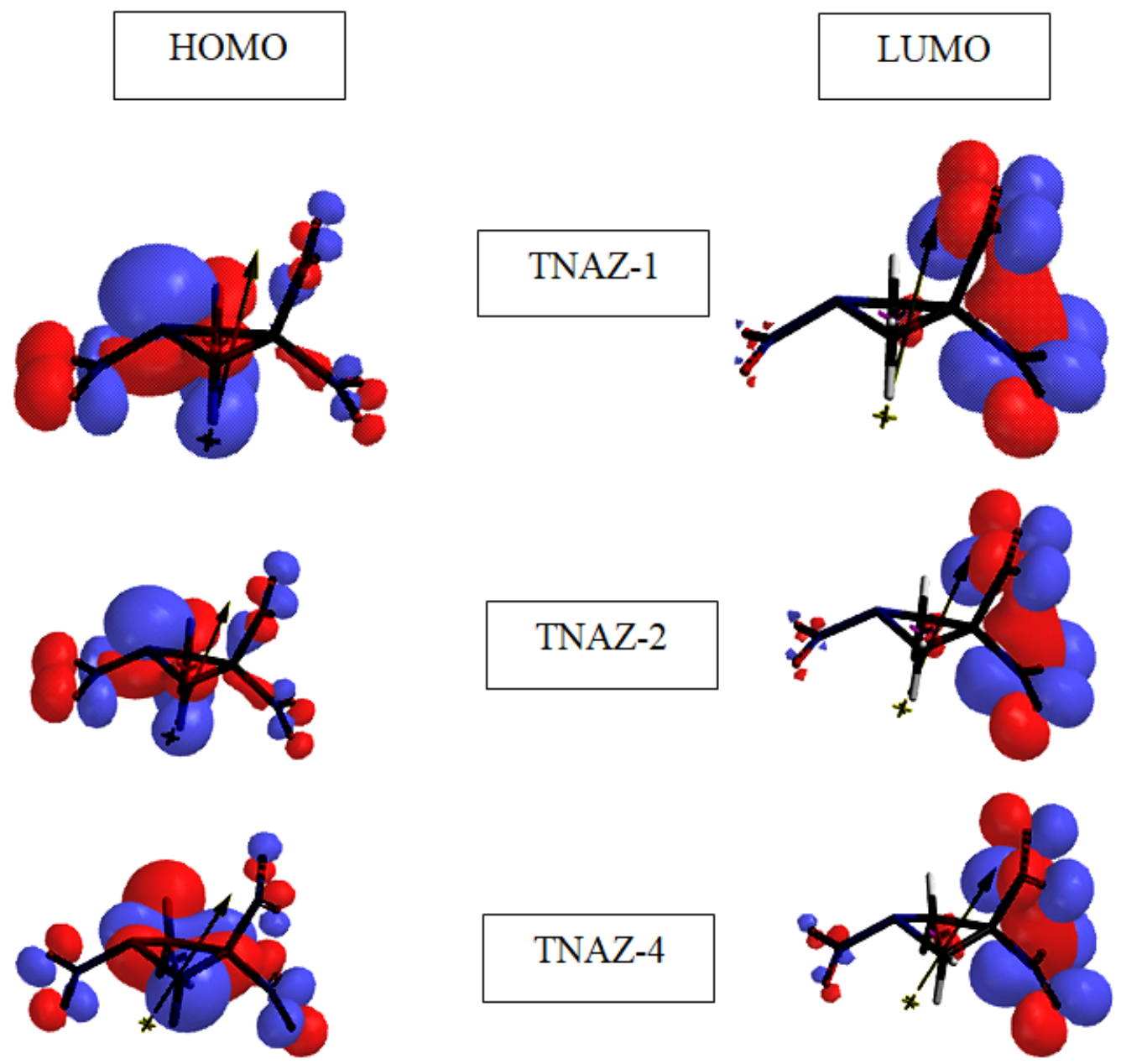

Figure 11. The HOMO and LUMO of some of the compressed TNAZ systems (along the Y-direction). 

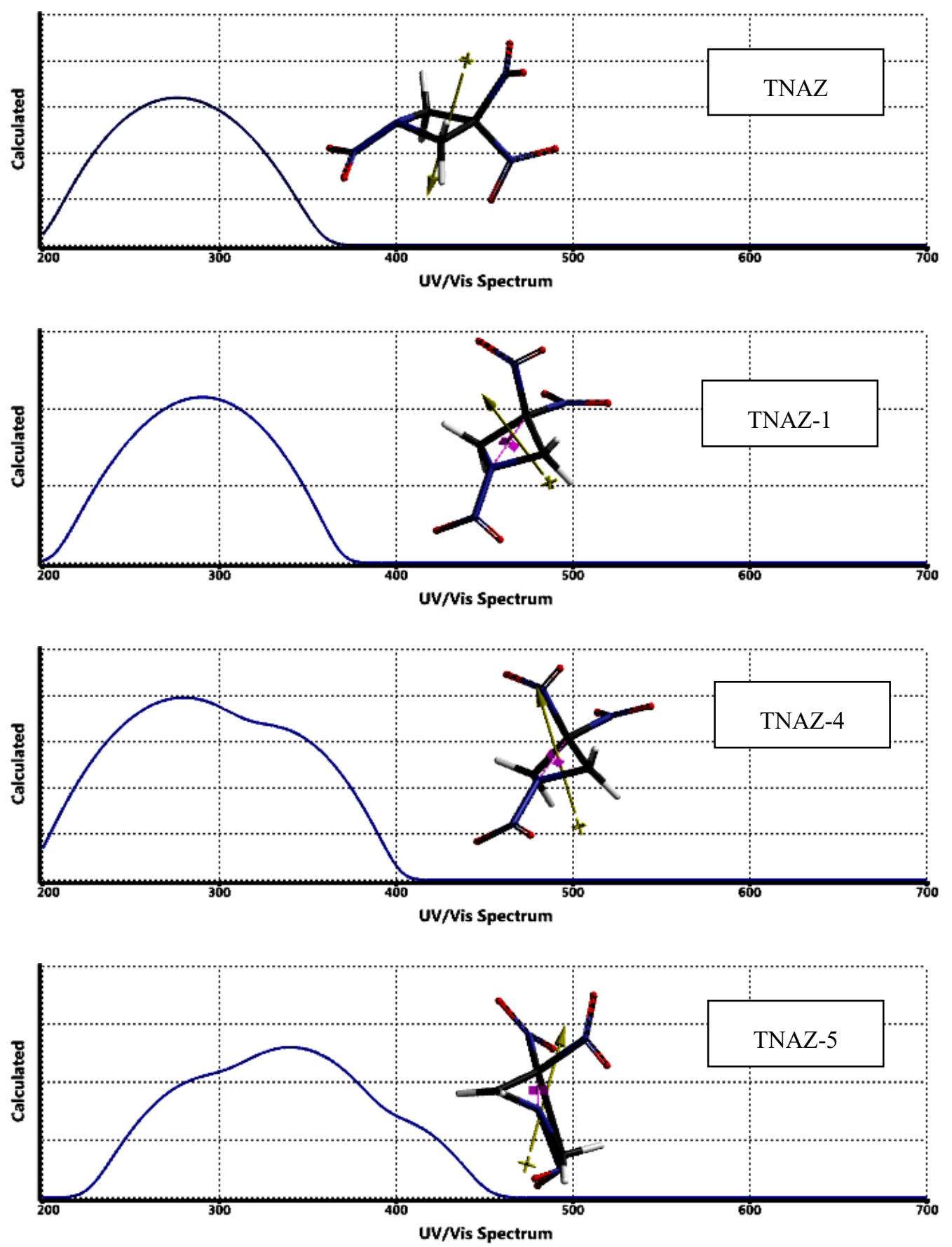

Figure 12. Calculated UV-VIS spectra of some compressed (along the X-direction) TNAZ systems. 

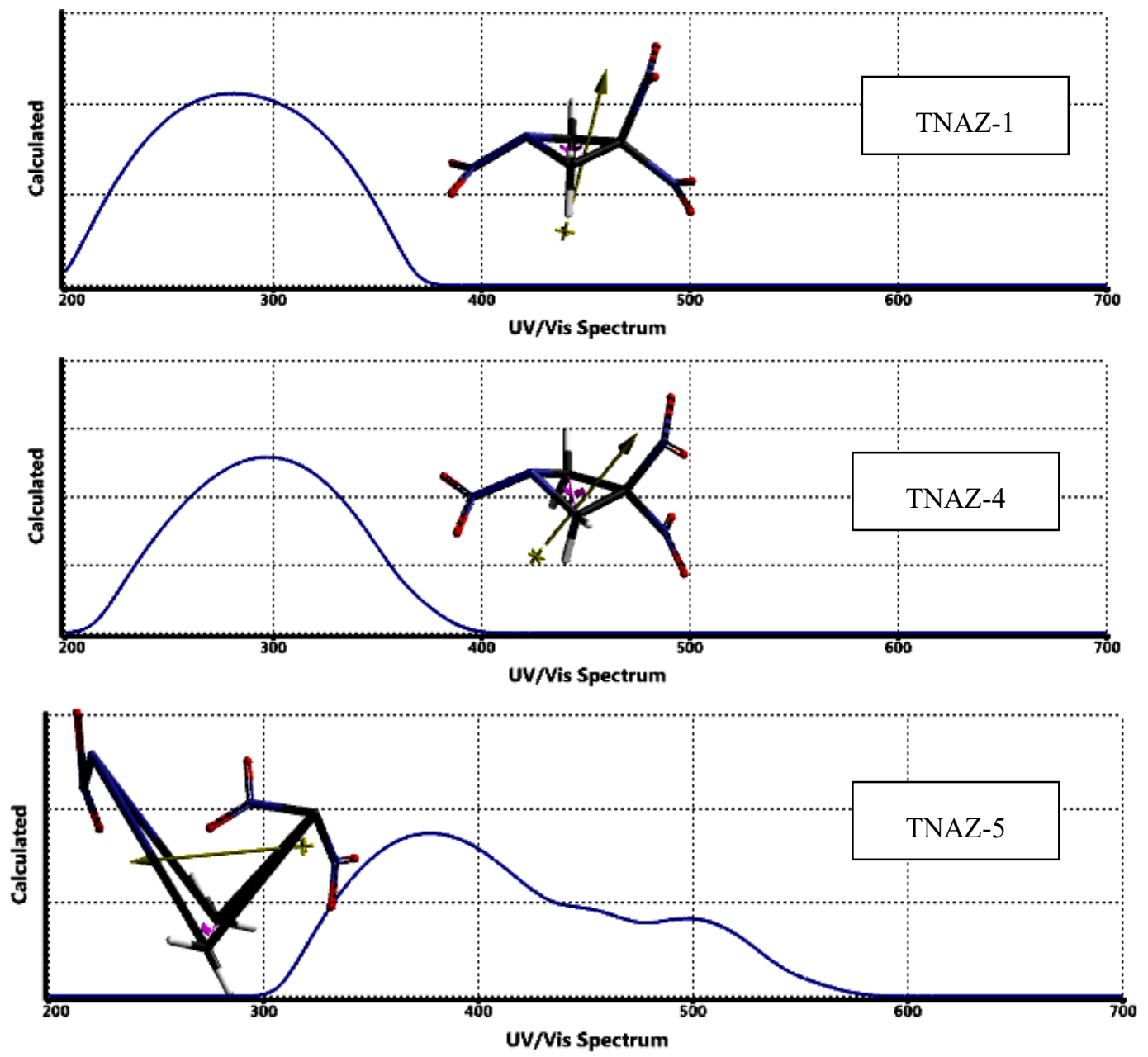

Figure 13. Calculated UV-VIS spectra of some compressed (along the Y-direction) TNAZ systems.

\section{Conclusion}

The present DFT treatment of the azetidine ring of TNAZ molecule, which is diagonally compressed along the X- or Y-direction at the molecular level, perturbs the structure generating various TNAZ-originated structures. Up to certain degree of compression TNAZ molecule remains intact but then breaks down. The compression along the X-direction eventually causes the rupture of $\mathrm{N}-\mathrm{NO}_{2}$ bond accompanied by the ring deformation. Whereas, the compression along the $\mathrm{Y}$-direction primarily results in a 
severe ring deformation accompanied by the conformational change of the nitro groups. The compression in both directions causes the narrowing of the interfrontier molecular orbital energy gap, thus should increase the impact sensitivity of TNAZ. However, the effect is more pronounced when the compression is applied in the Y-direction. As a result of narrowing of the interfrontier molecular orbital molecular orbital energy gap UV-VIS spectra show some bathochromic effect depending on the direction of the compression.

\section{References}

[1] P.F. Pagoria, G.S. Lee, R. A. Mitchell and R.D. Schmidt, A review of energetic materials synthesis, Thermochim. Acta 384 (2002), 187-204.

https://doi.org/10.1016/S0040-6031(01)00805-X

[2] H.S. Jadhav, M.B. Talawar, D.D. Dhavale, S.N. Asthana and V.V. Krishnamurthy, Alternate method to synthesis of 1,3,3-trinitroazetedine (TNAZ): Next generation melt castable high energy material, Indian J. Chemical Technology 13 (2006), 41-46.

[3] L. Türker, A composite of NTO and TNAZ - A DFT treatment, Earthline Journal of Chemical Sciences 5(2) (2021) 261-274.

https://doi.org/10.34198/ejcs.5221.261274

[4] L. Türker, A DFT treatment of some aluminized 1,3,3-trinitroazetidine (TNAZ) systems - A deeper look, Earthline Journal of Chemical Sciences 3(2) (2020), 121-140. https://doi.org/10.34198/ejcs.3220.121140

[5] T.G. Archibald, R. Gilardi, K. Baum and C.J. George, Synthesis and x-ray crystal structure of 1,3,3-trinitroazetidine, J. Org. Chem. 55 (1990), 2920-2924. https://doi.org/10.1021/jo00296a066

[6] R.L. McKenney, Jr., T.G. Floyd, W.E. Stevens, T.G. Archibald, A.P. Marchand, G.V.M. Sharma and S.G. Bott, Synthesis and thermal properties of 1,3-dinitro-3(1',3'-dinitroazetidin-3'-yl) azetidine (TNDAZ) and its admixtures with 1,3,3trinitroazetidine (TNAZ), J. Energ. Mater. 16 (1998), 199-235.

https://doi.org/10.1080/07370659808217513

[7] A.M. Hiskey, M.C. Johnson and E.D. Chavez, Preparation of 1-substituted-3,3dinitroazetidines, J. Energ. Mater. 17 (1999), 233-252.

https://doi.org/10.1080/07370659908216106 
[8] J. Zhang, R. Hu, C. Zhu, G. Feng and Q. Long, Thermal behavior of 1,3,3trinitroazetidine, Thermochim. Acta 298 (1997), 31-35. https://doi.org/10.1016/S0040-6031(97)00056-7

[9] S. Zeman, The thermoanalytical study of some amino derivatives of 1,3,5trinitrobenzene, Thermochim. Acta 216 (1993), 157-168. https://doi.org/10.1016/0040-6031(93)80389-R

[10] M.H. Keshavarz, Approximate prediction of melting point of nitramines, nitrate esters, nitrate salts and nitroaliphatics energetic compounds, J. Hazard. Mater. 138 (2006), 448-451. https://doi.org/10.1016/j.jhazmat.2006.05.097

[11] Z. Jalovy, S. Zeman, M. Suceska, P. Vavra, K. Dudek and J.M. Rajic, 1,3,3Trinitroazetidine (TNAZ). Part I. Syntheses and properties, J. Energ. Mater. 19 (2001), 219-239. https://doi.org/10.1080/07370650108216127

[12] D.S. Watt and M.D. Cliff, Evaluation of 1,3,3-trinitroazetidine (TNAZ) - A high performance melt-castable explosive, Technical Report DSTO-TR-1000, Defence Science and Technology Organization (DSTO), Aeronautical and Maritime Research Laboratory, Melbourne, Australia, 2000.

[13] A.K. Sikder and N. Sikder, A review of advanced high performance, insensitive and thermally stable energetic materials emerging for military and space applications, J. Hazard. Mater. 112 (2004), 1-15.

https://doi.org/10.1016/j.jhazmat.2004.04.003

[14] S. Iyer, E. Y. Sarah, M. Yoyee, R. Perz, J. Alster and D. Stoc, TNAZ based composition C-4 development, 11th Annual Working Group, Institute on Synthesis of High Density Materials (Proc.), Kiamesha Lakes, 1992.

[15] M. Oftadeh, M. Hamadanian, M. Radhoosh and M.H. Keshavarz, DFT molecular orbital calculations of initial step in decomposition pathways of TNAZ and some of its derivatives with $-\mathrm{F},-\mathrm{CN}$ and - $\mathrm{OCH} 3$ groups, Computational and Theoretical Chemistry 964 (2011), 262-268.

https://doi.org/10.1016/j.comptc.2011.01.007

[16] J.O. Doali, R.A. Fifer, D.I. Kruzezynski and B.J. Nelson, The mobile combustion diagnostic fixture and its application to the study of propellant combustion Part-I. Investigation of the low pressure combustion of LOVA XM-39 Propellant, Technical Report No: BRLMR-3787/5, US Ballistic Research Laboratory, Maryland, 1989. 
[17] J.P. Agrawal, Recent trends in high-energy materials, Prog. Energ. Combust. Sci. 24/1 (1998), 1-30. https://doi.org/10.1016/S0360-1285(97)00015-4

[18] M.D. Coburn, M.A. Hiskey and T.G. Archibald, Scale-up and wasteminimization of the Los Alamos process for 1,3,3-trinitroazetidine (TNAZ), Waste Management 17 (1997), 143-146.

https://doi.org/10.1016/S0956-053X(97)10013-7

[19] L. Jizhen, F. Xuezhong, F. Xiping, Z. Fengqi and H. Rongzu, Compatibility study of 1,3,3-trinitroazetidine with some energetic components and inert materials, Journal of Thermal Analysis and Calorimetry 85(3) (2006), 779-784. https://doi.org/10.1007/s10973-005-7370-8

[20] L. Türker and S. Varis, Desensitization of TNAZ via molecular structure modification and explosive properties - A DFT study, Acta Chim. Slov. 59 (2012), 749-759.

[21] J. Wu, Y. Huang, L. Yang, D. Geng, F. Wang, H. Wang and L. Chen, Reactive molecular dynamics simulations of the thermal decomposition mechanism of 1,3,3-trinitroazetidine, Chem. Phys. Chem. 19(20) (2018), 2683-2695. https://doi.org/10.1002/cphc.201800550

[22] J.J.P. Stewart, Optimization of parameters for semiempirical methods I. Method, J. Comput. Chem. 10 (1989), 209-220. https://doi.org/10.1002/jcc.540100208

[23] J.J.P. Stewart, Optimization of parameters for semi empirical methods II. Application, J. Comput. Chem. 10 (1989), 221-264. https://doi.org/10.1002/jcc.540100209

[24] A. R. Leach, Molecular Modeling, Essex: Longman, 1997.

[25] P. Fletcher, Practical Methods of Optimization, New York: Wiley, 1990.

[26] W. Kohn and L. Sham, Self-consistent equations including exchange and correlation effects, J. Phys. Rev. 140 (1965), A1133-A1138.

https://doi.org/10.1103/PhysRev.140.A1133

[27] R.G. Parr and W. Yang, Density Functional Theory of Atoms and Molecules, London: Oxford University Press, 1989.

[28] C.J. Cramer, Essentials of Computational Chemistry, Chichester, West Sussex: Wiley, 2004. 
[29] A.D. Becke, Density-functional exchange-energy approximation with correct asymptotic behavior, Phys. Rev. A 38 (1988), 3098-3100. https://doi.org/10.1103/PhysRevA.38.3098

[30] S.H. Vosko, L. Wilk and M. Nusair, Accurate spin-dependent electron liquid correlation energies for local spin density calculations: a critical analysis, Can. J. Phys. 58 (1980), 1200-1211. https://doi.org/10.1139/p80-159

[31] C. Lee, W. Yang and R.G. Parr, Development of the Colle-Salvetti correlationenergy formula into a functional of the electron density, Phys. Rev. B 37 (1988), 785-789. https://doi.org/10.1103/PhysRevB.37.785

[32] SPARTAN 06, Wavefunction Inc., Irvine CA, USA, 2006.

[33] A. Streitwieser, Jr., Molecular Orbital Theory for Organic Chemists, New York: Wiley, 1961.

[34] V. Anbu, K.A. Vijayalakshmi, R. Karunathan, A.D. Stephen and P.V. Nidhin, Explosives properties of high energetic trinitrophenyl nitramide molecules: A DFT and AIM analysis, Arab. J. Chem. 12(5) (2019), 621-632.

https://doi.org/10.1016/j.arabjc.2016.09.023

[35] N.R. Badders, C. Wei, A.A. Aldeeb, W.J. Rogers and M.S. Mannan, Predicting the impact sensitivities of polynitro compounds using quantum chemical descriptors, J. Energ. Mater. 24 (2006), 17-33. https://doi.org/10.1080/07370650500374326 